\title{
Proliferative retinopathy and neovascularization of the anterior segment in female type 2 diabetic rats
}

\author{
Jorge E Mancini ${ }^{1}$, Juan $O$ Croxatto $^{2}$ and Juan E Gallo ${ }^{1 *}$
}

\begin{abstract}
Background: To examine the presence of diabetic retinopathy in a female rat model of type 2 diabetes fed on a high-fat diet (HFD).

Methods: Wistar rats were injected with streptozotocin (STZ) at the age of two days and fed on an HFD from eight weeks onwards. Five diabetic animals were euthanized at 110 weeks of disease, together with a control group of age-matched, non-diabetic animals. A group of diabetic animals at 57 weeks of disease was included for comparison. Cross sections of the rats' corneas, iris and retinas were histologically examined and analysed by immunohistochemistry and immunofluorescence, using glial-fibrillary-acidic-protein (GFAP), the vascular endothelial growth factor (VEGF) and the Von Willebrand factor (VWF). The trypsine digestive technique was used for the pericytes count.

Results: Neovascularization was only found in the retinas, irises and corneas of the diabetic animals of 110 weeks of disease. There was also a significantly lower number of pericytes in these animals than in the controls.

Conclusion: The female rat model of type 2 diabetes fed on an HFD may prove useful in evaluating the mechanisms involved in diabetic retinopathy, together with strategies to reduce its severity.
\end{abstract}

Keywords: Type 2 diabetes, Diabetic retinopathy, Diet, Neovascularization, Animal model

\section{Background}

The prevalence of diabetes is increasing worldwide [1,2]. Factors involved in this special epidemia include a sedentary lifestyle, a fatty diet and being overweight. As a result, secondary diabetic complications have also risen [2]. Diabetic retinopathy is still the main cause of blindness in adults, despite important therapeutic advances.

The type 1 diabetes rat model has been widely used by visual scientists to analyse molecular mechanisms associated with diabetic retinopathy [3]. These animals are extremely fragile and die early, before retinal angiogenesis occurs. However proliferative retinopathy has not yet been reported in type 2 diabetic rats, who have a longer and healthier life [3-5]. This motivated investigators to seek new rat models of type 2 diabetes in which proliferative

\footnotetext{
* Correspondence: jgallo06@gmail.com

'Nanomedicine \& Vision Group, Facultad de Ciencias Biomedicas, Department of Ophthalmology, Hospital Universitario Austral, Universidad Austral, Pilar, Buenos Aires, Argentina

Full list of author information is available at the end of the article
}

changes could be studied. It is worth mentioning that choroidal and retinal neovascularization have been described in rat models without diabetes $[3,6]$, unveiling mechanisms similar to those reported in proliferative diabetic retinopathy [7].

In a previous study, we aimed to develop a proliferative diabetic retinopathy animal model in male type 2 diabetic rats fed on a high-fat diet (HFD) with a followup of 90 weeks. However, no proliferative changes were found (unpublished data). For this reason, we decided to conduct a new and longer study. This time the investigation was performed in a group of female rats, due to recent reports that higher levels of hyperglycaemia have been observed in female than male rats $[8,9]$. Female rats may develop much more severe diabetes $[8,10]$. In humans, recent investigations carried out in postmenopausal women reported an inverse relationship between serum concentrations of sex hormone binding globulin (SHBG) and the risk of clinical diabetes [11,12]. It is also known that oestrogens enhance angiogenesis under
C Biomed Central 
special circumstances, as in cancer diseases [13,14]. However, it should be noted that a difference in diabetes growth between male and female rats is still a controversial subject.

This paper reports the outcome of a research study carried out on female type 2 diabetic rats fed on an HFD, with a follow-up of 110 weeks (two years), that developed proliferative changes in the retina, iris and cornea. To our knowledge, this is the first report to demonstrate the presence of proliferative diabetic retinopathy and neovascularization of the anterior segment in rats.

\section{Methods}

\section{Rat model of diabetes}

Pregnant Wistar Rats (provided by CONEA, Buenos Aires) were housed in the animal facilities section of the School of Biomedical Sciences of Austral University, at $21 \pm 1^{\circ} \mathrm{C}$ on a 12 -h light-dark cycle. They were examined daily until delivery (at 9 am and $6 \mathrm{pm}$ ). Two days after birth, the newborn rats were intraperitoneally injected with streptozotocin (STZ) (45 mg/kg) (Sigma Lab), in $0.1 \mathrm{ml}$ of a $0.1 \mathrm{M}$ solution of citrate buffer of $154 \mathrm{~mm}$ of $\mathrm{NaCl}$ at $4.5 \mathrm{pH}[9,15,16]$. The rat pups remained with the mothers until they were 21-days-old. Eight weeks later, the STZ-treated animals were fed with a home-made HFD. Table 1 shows the composition of the diet, which was prepared every week and stored at $-7^{\circ} \mathrm{C}$. Glycaemia levels were measured one week after starting the diet and every month thereafter. Blood samples of $32 \mu \mathrm{l}$ were collected by tail snipping and tested using the Reflotron System Boehringer Mannheim. High levels of glycaemia $(160-220 \mathrm{mg} / \mathrm{dl})$ were found in rats fed on the HFD [17].

Table 1 The composition of the high-fat diet (HFD) and standard diet

\begin{tabular}{|c|c|c|c|c|c|}
\hline & & \multicolumn{2}{|l|}{ HFD } & \multicolumn{2}{|c|}{ Standard } \\
\hline & & Total & Subtotal & Total & Subtotal \\
\hline Carbohydrates & & $28.8 \%$ & & $54.2 \%$ & \\
\hline Protein & & $23.6 \%$ & & $23.6 \%$ & \\
\hline \multirow[t]{4}{*}{ Amino acids } & & $2.2 \%$ & & $2.2 \%$ & \\
\hline & Lysine & & $51.1 \%$ & & $51.1 \%$ \\
\hline & Methionine & & $26.6 \%$ & & $26.6 \%$ \\
\hline & Threonine & & $22.2 \%$ & & $22.2 \%$ \\
\hline \multirow[t]{4}{*}{ Fat } & & $25.6 \%$ & & $2.7 \%$ & \\
\hline & Saturates & & $46.4 \%$ & & $24.2 \%$ \\
\hline & Monounsaturated & & $47.5 \%$ & & $30.2 \%$ \\
\hline & Polyunsaturated & & $6.3 \%$ & & $45.6 \%$ \\
\hline \multirow[t]{3}{*}{ lons } & Calcium & $1.6 \%$ & & $1.4 \%$ & \\
\hline & Sodium & $0.05 \%$ & & $0.05 \%$ & \\
\hline & Magnesium & $0.18 \%$ & & $0.18 \%$ & \\
\hline
\end{tabular}

Two groups of twelve and five animals were euthanised at 57 and 110 weeks of diabetes, respectively.

\section{Control rats}

Non-diabetic animals with no STZ injection were fed on standard food with a $2.71 \%$ fat content (Table 1 ). Their levels of glycaemia were normal $(80-95 \mathrm{mg} / \mathrm{dl})$. Twelve controls animals were euthanized at 62 weeks old and five at 115 weeks old.

Animals used in the study were handled according to the Association of Research in Vision and Ophthalmology (ARVO) Statement for the use of animals in ophthalmic research.

\section{Clinical parameters}

Each animal was weighed using a standard scale at eight weeks of life and before death. Lipidaemia levels were determined using the Reflotron System every month and before the animal was euthanised.

\section{Fatty acid profile}

The chemical compound of the HFD and conventional diets are shown in Table 1 . In brief, the HFD contained $25.6 \%$ of fat $(46.4 \%$ saturated, $47.5 \%$ monosaturated and $6.3 \%$ polysaturated) while the conventional diet contained $2.71 \%$ of fat $(24.2 \%$ saturated, $30.2 \%$ monosaturated and $45.6 \%$ polysaturated). The diet's chemical compound was analysed using animal and vegetable fats and oil-analysis by gas chromatography of methyl esters of fatty acids according to The International Organization for Standardization (ISO 5508: 1990-E).

\section{Clinical photographs and gross sections}

Clinical photographs were taken of the animals when they were still alive and before anaesthesia. For the gross sections, we removed the ocular globe, then used a $30 \mathrm{G}$ needle to inject an air bubble through the corneal limbus into the anterior chamber to make the presence of blood vessels in the cornea more evident. Both pictures were taken with a NIKON digital camera DS3000 (Tokyo, Japan) using the macro function.

\section{Histological examination}

The rats were anaesthetised using $350 \mathrm{mg} / \mathrm{kg}$ of chloral hydrate, delivered by an intraperitoneal injection. The eyes were removed and fixed in $4 \%$ paraformaldehyde (Sigma-Aldrich, St Louis, MO). Rats were then euthanised using an overdose of chloral hydrate. The eyes were left for one day for fixation, then immersed for cryoprotection in four concentrations of glucose (5\% overnight and $7.5 \%, 10 \%$ and $20 \%$ for two hours each) and interlocked with resin. Ten-micron cryosections were obtained (Shandon AS325 Retraction) and stained with haematoxylin and eosin $(\mathrm{H} \& \mathrm{E})$ as well as periodic 
Table 2 Weight, glycaemia, trygliceridaemia and cholesterolaemia in diabetic and control rats

\begin{tabular}{|c|c|c|c|c|c|c|c|}
\hline \multirow[t]{2}{*}{ Group } & \multirow[t]{2}{*}{ No. rats } & \multirow[t]{2}{*}{ WA } & \multirow[t]{2}{*}{ WD } & \multicolumn{4}{|c|}{ Parameters *(SD) } \\
\hline & & & & Weight** & $\mathrm{Gl}^{* * *}$ & Tri*** & $C h^{* * *}$ \\
\hline CONTROL & 12 & 8 & - & $320(30)$ & $99.7(31.9)$ & $\mathrm{N}-\mathrm{M}$ & $\mathrm{N}-\mathrm{M}$ \\
\hline DBT-HFD & 12 & 8 & 0 & $340(28)$ & $125(21)$ & $\mathrm{N}-\mathrm{M}$ & $\mathrm{N}-\mathrm{M}$ \\
\hline CONTROL & 12 & 62 & - & $524(80)$ & $86(5.2)$ & 91 & 119 \\
\hline DBT-HFD & 12 & 65 & 57 & $449(76)$ & $158(22)$ & 114 & 115 \\
\hline CONTROL & 5 & 115 & - & $266(45)$ & $100(18.8)$ & 113 & 118.5 \\
\hline DBT-HFD & 5 & 118 & 110 & $279(62)$ & $232(81)$ & 122 & 115 \\
\hline
\end{tabular}

N-M: not measured; WD: weeks on HFD; WA: weeks of age; Gl: fasting glycemia; Tri: triglyceridaemia; Ch: cholesterolaemia; * Mean (standard deviation); ${ }^{* *} \mathrm{gr} ;{ }^{* * *} \mathrm{mg} / \mathrm{dl}$.

Diabetic animals with fasting glycaemia $<160 \mathrm{mg} / \mathrm{dl}$ were excluded. Control animals with fasting glycaemia $>130 \mathrm{mg} / \mathrm{dl}$ were not included in the study.
acid-Schiff stain (PAS) for microscopic examination using an Eclipse Nikon E800 Microscope (Tokyo, Japan). At least five sections per eye were examined.

\section{Immunohistochemical and immunofluorescent analyses}

The eyes were removed and fixed for 48 hours in $4 \%$ paraformaldehyde (Sigma-Aldrich, St Louis, MO). They were then immersed for cryoprotection in four concentrations of glucose $(5 \%$ overnight and $7.5 \%, 10 \%$ and $20 \%$ for two hours each) and interlocked with resin. Ten-micron sections were obtained and fixed on polylisine-treated glass slides (Shandon AS325 Retraction).

For immunohistochemistry, the sections were first incubated in biotinylated goat anti-mouse IgG, then in an avidin-biotin peroxidase complex kit and finally in 3.3'diaminobenzidine (DAB)/nickel solution. For immunofluorescence, axial sections were revealed using the secondary antibody goat-anti mouse with fluorescein.

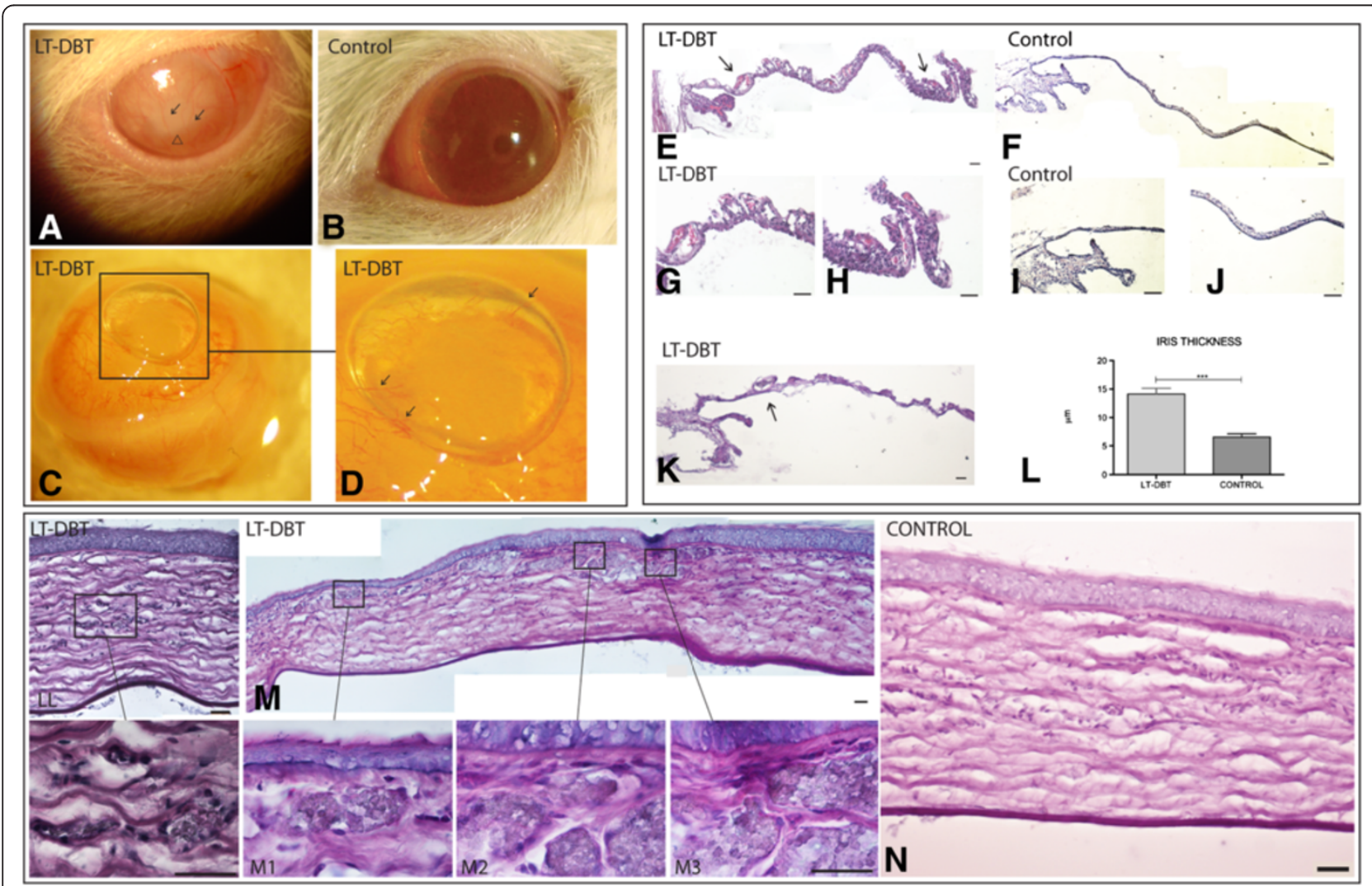

Figure 1 Anterior segment vessels. A: picture of in vivo 110-week-old animal (LT-DBT). The eye shows vessels in the cornea (arrow) and cataract (arrow head). B: in vivo 110-week-old control animal. C: gross section from an enucleated LT-DBT eye with an air bubble in the anterior chamber. D: Magnification from the $C$ photograph showing the presence of vessels in the cornea (arrow). Light microscopy, PAS and eosin $(\mathbf{E}, \mathbf{G}, \mathbf{H}, \mathbf{K})$ and haematoxylin and eosin $(\mathbf{F}, \mathbf{I}, \mathbf{J})$ iris staining from a LT-DBT animal $(\mathbf{E}, \mathbf{G}, \mathbf{H}, \mathbf{K})$. Arrows indicate the presence of abnormal vessels in the iris. $\mathbf{F}, \mathbf{I}$ and J: control iris from a non-diabetic age-matched rat. Bar $20 \mu \mathrm{m}$. L: The iris thickness is higher in the LT-DBT rats represented in $\mu \mathrm{m}$. ${ }^{* *} \mathrm{p}<0.0001$, LT-DBT mean $14.11 \pm 1.010(\mathrm{~N}=50)$, control mean $6.557 \pm 0.5647(\mathrm{~N}=47)$. Light microscopy; haematoxylin and eosin and PAS and eosin stained corneas. LL and $\mathbf{M}$ : vessels in the corneal stroma of LT-DBT animals, LL1 and M1, 2 and 3 are magnification of vessels within the corneal stroma. N: normal control cornea from a LT-Control rat. Bar $20 \mu \mathrm{m}$. 
Immunofluorescent analysis was carried out using the Eclipse Nikon Microscope (Tokyo, Japan).

GFAP expression was analysed using the primary monoclonal antibody anti-GFAP (BIOGENEX, 4600 Norris Canyon Road, San Ramon, CA, USA).

The immunoreactivity of the vascular endothelial growth factor (VEGF) was examined using the anti-VEGF polyclonal antibody (Santa Cruz Biotechnology Inc, 2145 Delaware Avenue, Santa Cruz, CA) and the anti humanvWF (Sigma-Aldrich, St Louis, MO: f3520) was used for the Von Willebrand factor (vWF).

\section{Trypsin digestive technique}

After the cornea was incised, the eyeball was fixed by immersing it, for a minimum of four hours, in $4 \%$ formalin buffered with $50 \mathrm{mM}$ Na-K phosphate (pH 7.2). The retina was dissected and then placed in the $4 \%$ buffered formalin for a further hour. The retina was cut into a segment adequate for handling and washed overnight in running water. After that, it was incubated at $37^{\circ} \mathrm{C}$ in a solution of $3 \%$ trypsin (Difco 1:250) and $0.1 \mathrm{M}$ tris buffer $(\mathrm{pH}$ 7.8) for one to three hours. The incubation was finished when the medium became cloudy and the tissue showed signs of digestion. The internal limiting membrane was peeled off in one sheet $[18,19]$. The network of vessels was freed of adherent retinal tissue by gentle shaking, mounted on a clean slide and allowed to dry. The preparation was stained with PAS and eosin.

\section{Image analysis: count of pericytes}

Image analysis measurements were made using a KS400 system (Kontron Elektronic/Zeiss, Eching, Germany) and a Nikon DXM 1200 digital camera (Tokyo, Japan) mounted onto an Eclipse Nikon E-800 microscope for image acquisition. Briefly, images were digitalised in a rectangular frame of $1280 \times 960$ pixels using the 40X objective in the photo mode of illumination intensity. To adjust for possible defects in the illumination of the optical pathway, a low-pass image was produced for subtraction and background shading correction. After that, a grey value for image segmentation was interactively chosen. In order to define a threshold grey level, all the pixels whose grey value informative content was lower or higher than the segmentation grey were set to white and the others were set to black. The pericytes were counted in ten randomly-selected sections of each retina by one observer.
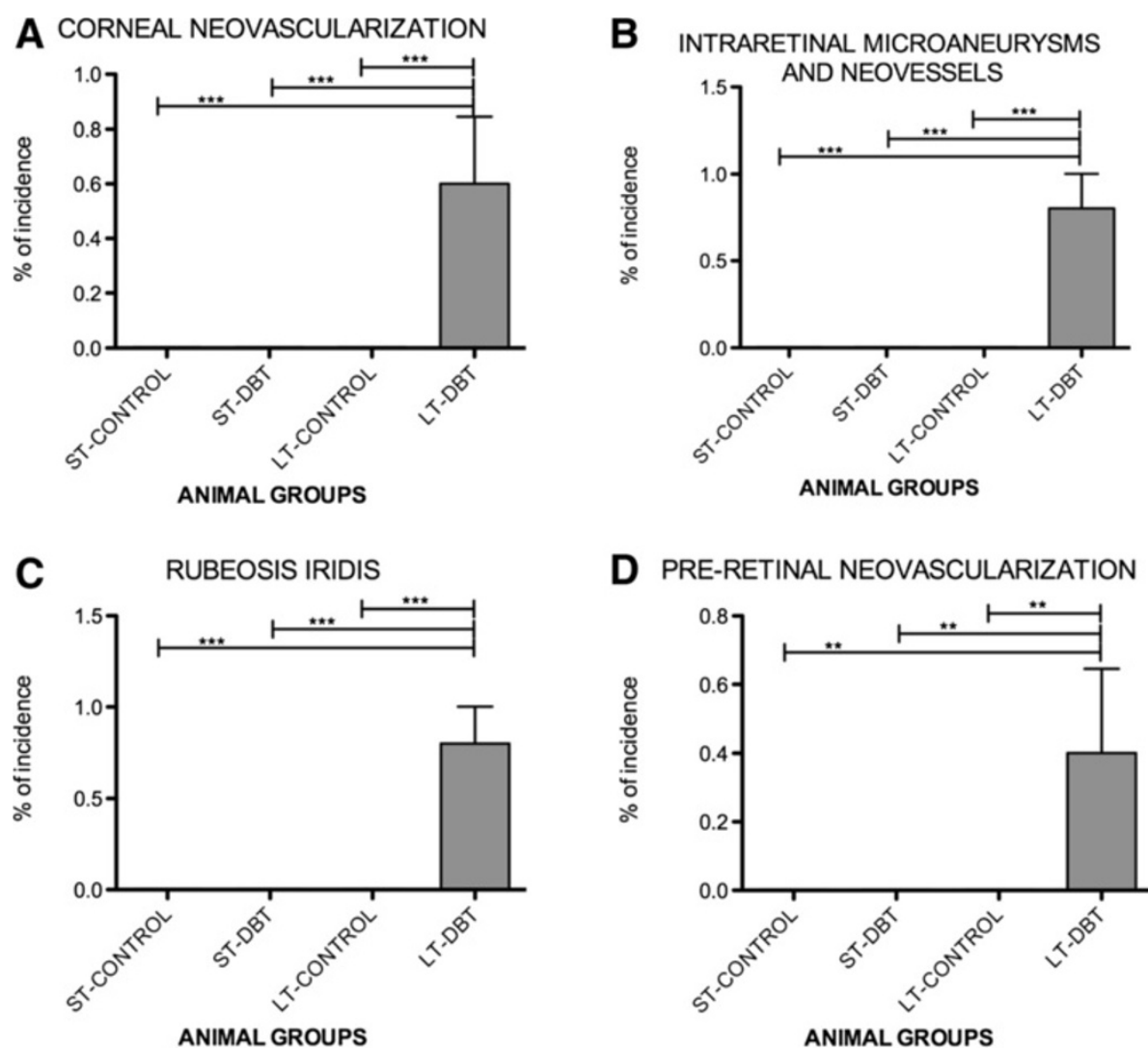

D PRE-RETINAL NEOVASCULARIZATION

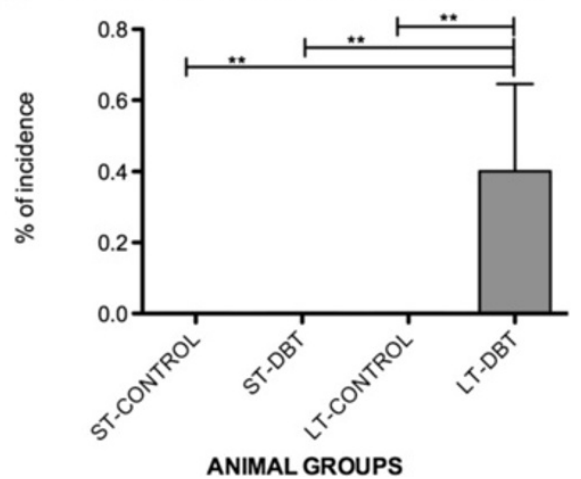

Figure 2 Incidence: statistical analyses; the one-way ANOVA (and nonparametrics) was used in all the cases with the Newman-Keuls multiple comparisons post test. (A) represents the corneal neovascularization, (B) intraretinal microaneurysms and neovessels, (C) rubeosis iridis, (D) presence of pre-retinal neovascularization. $(p<0,01)$. 
The number of pericytes was normalised to the relative capillary density (number of cells per millimeter squared of capillary area -3 pixels $^{2}$ of capillary area $=$ $\left.1 \mathrm{~mm}^{2}\right)$. The mean value was calculated in each animal. Samples were evaluated in a masked fashion. Statistical analysis was carried out using T-test analysis. All elements outside of the two standard deviations (SD) were eliminated.

\section{Iris thickness measurement}

Thickness measurements of the iris were made in all Long-term diabetics animals (LT-DBT) and control animals of 110-weeks-old. We used ten samples (100x) of each iris from the pupil to the iris base, distributed between $300 \mu \mathrm{m}$ in each sample. Samples were evaluated in a masked fashion. The image analyses were done with Image-J programme (Image Processing and Analysis in Java, NIH) and were performed using a Nikon DXM 1200 digital camera (Tokyo, Japan) mounted onto an Eclipse Nikon E-800 microscope for image acquisition. Images were digitalised in a rectangular frame of $1280 \mathrm{x}$ 960 pixels using the 100x. To adjust for possible defects in the illumination of the optical pathway, a low-pass image was produced for subtraction and background shading correction. The statistical analysis was the same as we used for the pericytes.

\section{Results}

Diabetic rats had higher glycaemia levels (range 140$416 \mathrm{mg} / \mathrm{dl}$ and mean $232 \mathrm{mg} / \mathrm{dl}, \mathrm{SD}$ 81.9) at 110 weeks of diabetes compared to age-matched control rats (range $80-120 \mathrm{mg} / \mathrm{dl}$ and mean $100.5 \mathrm{mg} / \mathrm{dl}, \mathrm{SD} 18.8)$. Higher levels of triglyceridaemia were observed in the diabetic animals than the controls (Table 2). The chemical composition of the diet is shown in Table 1.

When we looked at the anterior segment, the clinical photographs and gross sections showed the presence of cataracts in the five LT-DBT (110 weeks old), in two out of five 110-week-old control rats and in none of the short-term diabetic rats (ST diabetics, 57 weeks old) and 62 -week-old control rats. The difference between LTDBT rats and the other animal groups was statistically significant $(\mathrm{p}<0.05)$ (Figure 1$)$. Corneal neovascularization was seen in three out of the five LT diabetics. Neovessels were observed in the periphery and centre of the cornea, with an extension of at least one quadrant in each animal (Figure 1: A,C and D). The irises of all LT diabetic animals were significantly thicker than the controls due to the presence of abnormal vessels (Figure 1: L) $(\mathrm{p}=0.0309$, pair t-test; $\mathrm{p}<0.0001$, LT-DBT mean $14.11 \mu \mathrm{m} \pm 1.010 \mathrm{~N}=50$, control mean $6.557 \mu \mathrm{m} \pm$ $0.5647 \mathrm{~N}=47$ ). At least five neovessels were found in each iris section (Figure 1: E-K). Corneal histological analysis
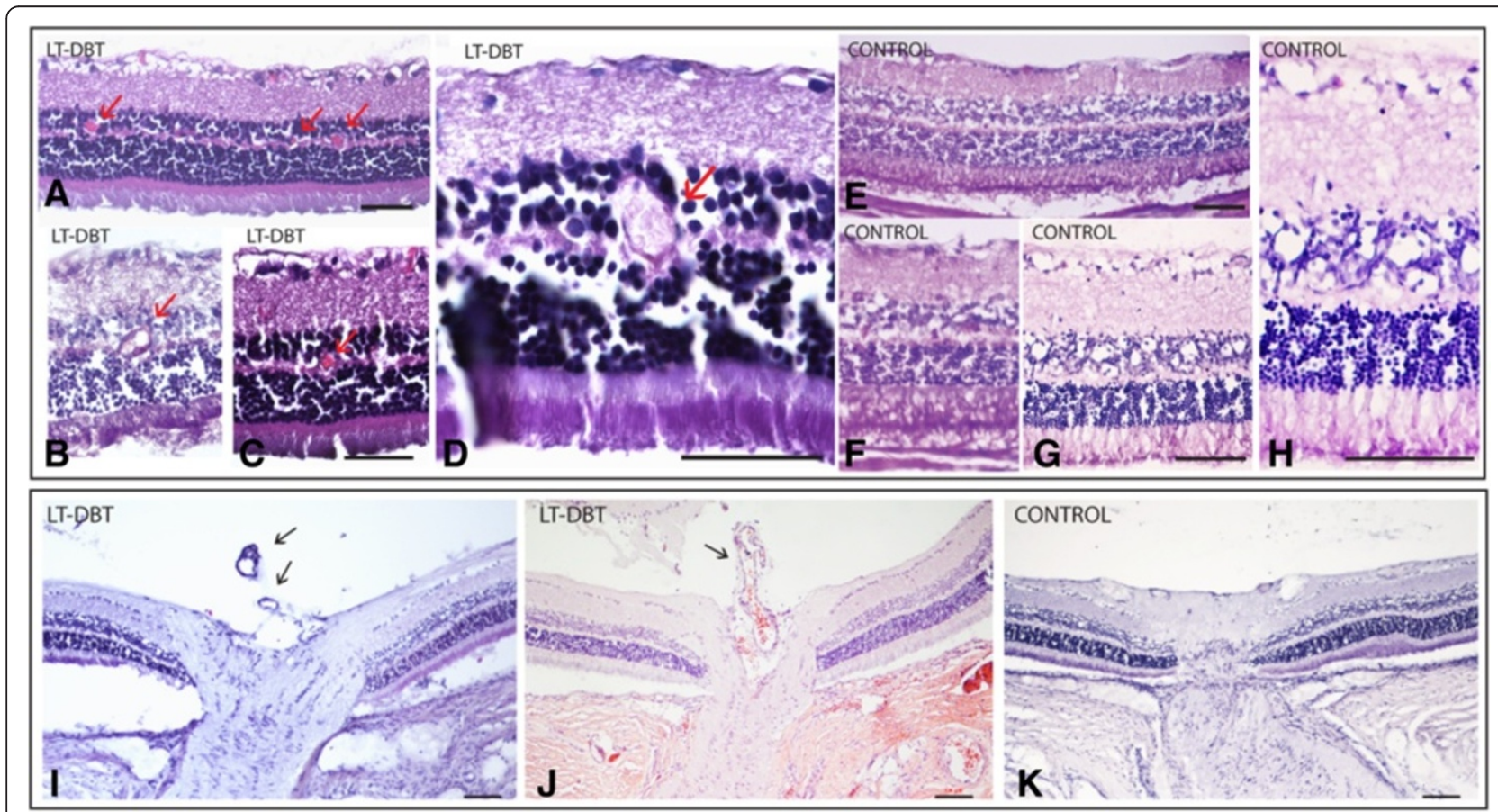

Figure 3 Posterior segment. Retina light microscopy: PAS and eosin (A and C) and haematoxylin and eosin (B, D, E, F, G and H) stained retinas. A, B, C and $\mathbf{D}$ are retinas from different LT-DBT animals. Arrows in picture $\mathbf{A}, \mathbf{B}, \mathbf{C}$ and $\mathbf{D}$ are pointing to vessel dilations in the outer plexiform layer. $\mathbf{E}, \mathbf{F}, \mathbf{G}$ and $\mathbf{H}$ show the LT-Control retina. Bar $20 \mu \mathrm{m}$. Optic nerve. Haematoxylin and eosin (I and $\mathbf{K})$ and PAS and eosin (J) staining from transverse sections of the optic nerve, I and $\mathbf{J}$ : LT-DBT animals with vessels from nerve to vitreous (arrows) and $\mathbf{K}$ : normal optic nerve from LT-Controls. Bar 20 um. 
showed neovessels in the epithelium and anterior stroma (Figure 1: LL and M and Figure 2). No neovessels in the cornea and iris were seen in either the controls or the ST diabetics (Figure 2).

When we looked at the posterior segment, we saw intraretinal neovessels in all LT diabetic animals (Figure 3). Vessel dilation or microaneurysms were found in four animals (Figure 2; Figure 3: A - D). None of these findings were seen among the controls or ST diabetics. Pre-retinal vessels were histologically seen near the optic nerve in two LT diabetics (Figure 2; Figure 3: I and J).

The immunoreactivity for VEGF and vWF in the retina, iris and cornea was analysed by anti-vWF antibodies (Figure 4, column 1) and anti-VEGF (Figure 4, column 2). We only observed up-regulation of vWF and VEGF in the
LT diabetics (Figure 4: A1, A2, D1, D2 and G1, G2). The retina showed up-regulation of vWF in vessels localised in the fibre layer (FL) and in the outer plexiform layer (OPL) (Figure 4: A1), while VEGF up-regulation was found in the OPL (Figure 4: A2). In this layer, co-localisation of vWF and VEGF was observed (Figure 4: A3). A similar coexpression of the two proteins (vWF and VEGF) was found in vessels along the iris stroma (Figure 4: D1, D2 and D3). In the cornea, the immunoreactivity for vWF and VEGF was observed in stromal vessels (Figure 4: G1, G2 and G3). Neither the ST diabetic group nor the controls exhibited immunoreactivity for VWF and VEGF in the retina, iris or cornea (Figure 4: rows $\mathrm{H}$ and I).

The number of pericytes measured by the trypsin digestive technique was found to be significantly lower in

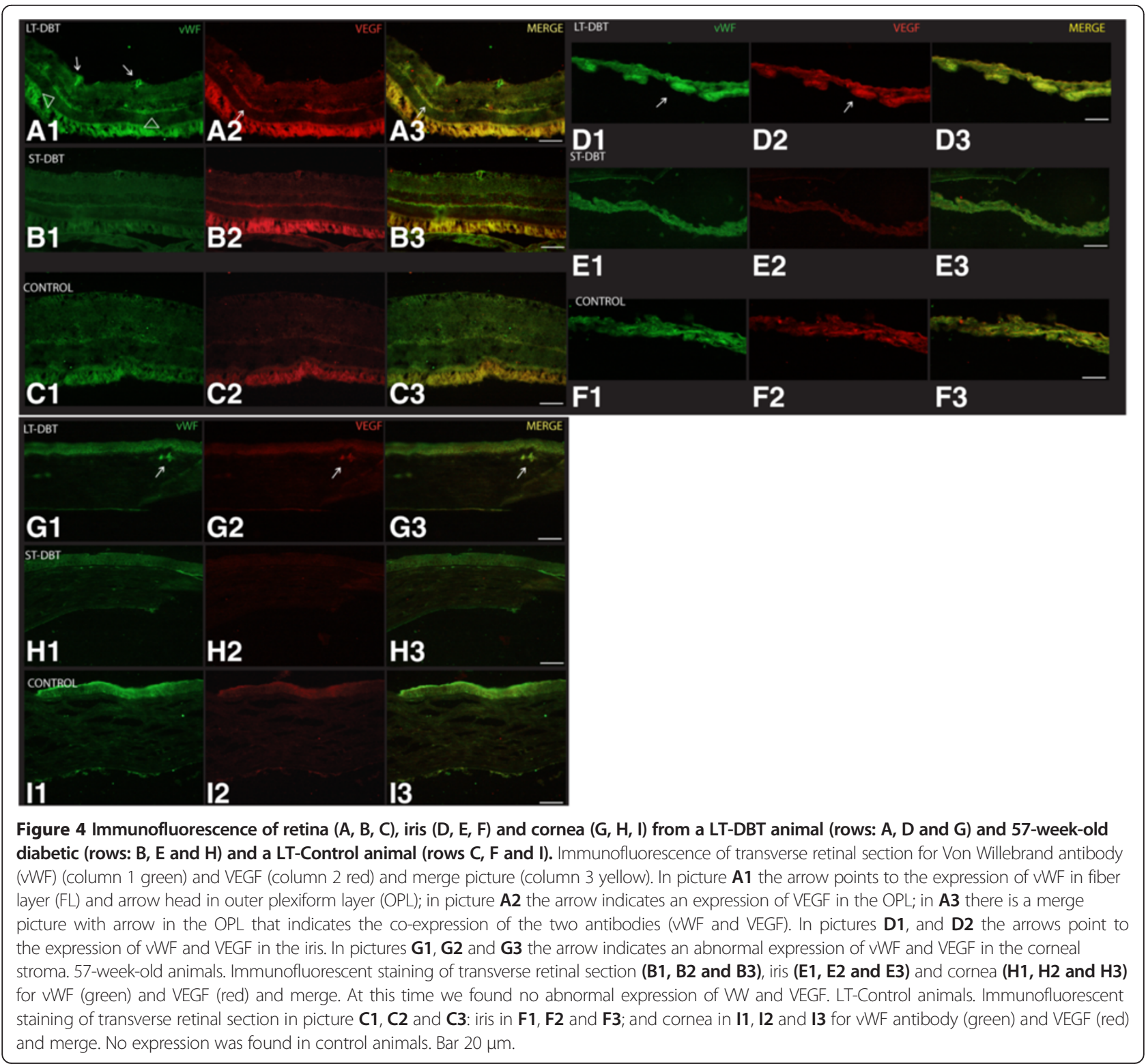


the LT-diabetic group than in the controls (Figure 5) $(\mathrm{p}=0.0298$ unpair $\mathrm{t}$-test $\mathrm{p}<0.05$; Mean \pm SEM of Control $0.08670 \pm 0.003975 \mathrm{~N}=31$; Mean \pm SEM of DBT group $0.07206 \pm 0.005373 \mathrm{~N}=21$ ). Positive immunoreactivity for GFAP was found in the FL and in cells morphologically similar to Müller cells. Staining was much more extensive in the LT diabetics than in the ST diabetics and controls (Figure 5).

\section{Discussion}

We carried out an experimental study of diabetic retinopathy in an animal model of female type 2 diabetic rats fed on an HFD. The animals developed retinal and corneal neovascularization, as well as rubeosis iridis, after 110 weeks (two years) of diabetes. To our knowledge, this is the first report on ocular neovascularization in type 2 diabetic rats fed on an HFD.

Animal models are extremely important for the advancement of science and from time to time researchers report new models of diseases. However, the complexity of some diseases makes this task difficult, particularly when both systemic and local factors contribute to the illness, as in the case of diabetic retinopathy. Another element to consider is the cost of animal models, which should be reasonable. Big animals are expensive and difficult for researchers to afford [4]. So, these aspects convinced us to opt for the rat model of type 2 diabetes fed on an HFD. This model was previously characterised by other investigators $[16,20]$ and recommended for studies on diabetic complications associated with obesity [21].

It should be mentioned that we did not find proliferative retinopathy changes in a previous study carried out in male type 2 diabetic rats of 90 weeks of disease, who were also fed on an HFD (unpublished data). Although the duration of diabetes is five months longer in this survey of female rats, other factors can lead to the growth of proliferative changes in these animals. The potential influence of oestrogens in the progression of diabetes in rats is known [22] and this is possibly enhanced by depletion of SHBG in female rats. It has been reported that female rats are less sensitive to insulin than males of all age groups and also more susceptible to the rapid development of a severe form of diabetes. This is still controversial in the scientific literature. In any case, the existence of sex receptors in the rat eye may play a role in retinopathy development. These receptors are also found in the human eye [23,24]. The chemical compound of the HFD used in our research studies in male and female diabetic rats was the same and we know that this type of diet increases the risk of cardiovascular disease and promotes cell damage [25-27]. So, an early onset of diabetes (STZ injection at day two, followed by HFD at eight weeks onwards), a longer duration, known clinical risk factors and female sex might have been key elements in the development of ocular neovascularization.

The first retinal morphological changes observed in diabetic rats described in the scientific literature included

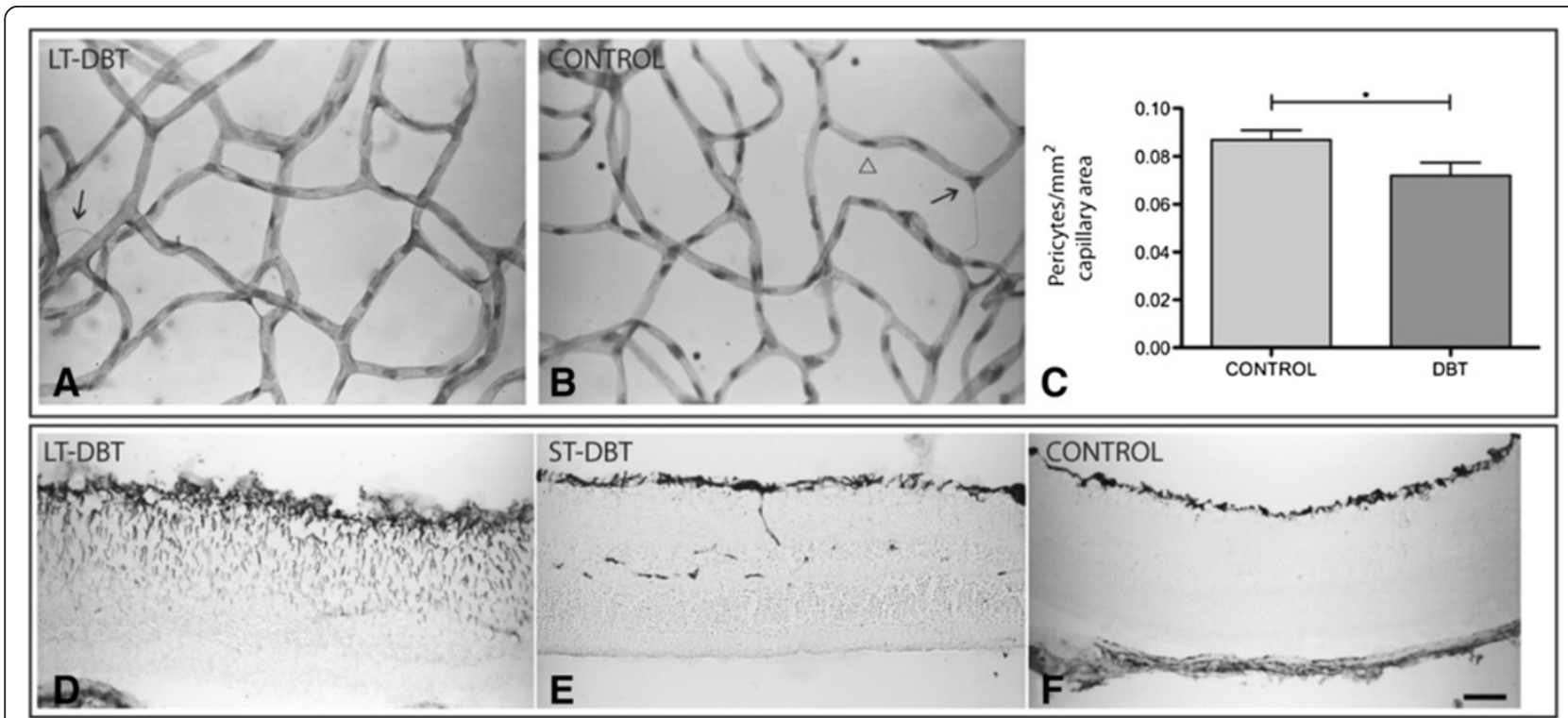

Figure $\mathbf{5}$ Additional studies. Tripsin digestive technique of the retina (A, B). A, LT-DBT capillary-net, arrows point to acellular capilary. B: vascular capillary-net from a retina between middle size vessels. Arrow points to pericytes and arrowhead to endothelial cells. C, Number of pericytes per capillary area was determined as indicated in methods section. Results are mean \pm SEM of five rats in each group. ${ }^{*} P<0.05$. $\mathbf{D}, \mathbf{E}$ and $\mathbf{F}$ are transverse retinal sections with GFAP immunostaining from LT-DBT rats (D), ST-DBT rats (E) and controls (F). Diabetic retinas (D and E) overexpressed GFAP in the fibre layer and in cells with the morphology and spatial organisation of Müller cells. Bar 50 m. 
loss of pericytes and the presence of acellular and collapsed capillaries. These changes lead to retinal ischaemia [28]. We found a significantly lower number of pericytes in LT diabetic rats than in the controls, which is in accordance with previous studies.

In our survey, the morphology of pre-retinal neovascularization was consistent with that reported by other authors $[3,29,30]$. Intra-retinal neovessels were seen in the inner part of the retina (Figure 2). This type of vessel always shows positive immunoreactivity of the vWF Factor and VEGF as well as co-localisation of them. It is known that $\mathrm{vWF}$ is a marker of endothelial dysfunction and widely used to study angiogenesis in cancer and ocular diseases $[31,32]$. Staining of this marker was not seen in the vessels of the ST diabetics and controls. It should be noted that all LT diabetic animals had pre-retinal and/or intra-retinal neovessels. Similarly, all LT diabetics showed a thick iris, as well as rubeosis iridis, a known consequence of severe retinal hypoxia and the release of angiogenic factors that lead to neovascular glaucoma in humans. This, in turn, can cause corneal endothelial dysfunction with bullous keratopathy, corneal edema and epithelial erosions, release of inflammatory cytokines, limbal stem cells deficiency and corneal neovascularization, which was clinically observed in three of the LT diabetic animals in our study.

\section{Conclusions}

An animal model mimics a human disease and it can be considered as a platform for the study of mechanisms and therapeutic agents. For this reason, several models have been developed in ophthalmology, such as the oxygeninduced retinopathy model [33] and the proliferative diabetic retinopathy model using gene therapy [30]. In our model, the streptozotocin injection, HFD, earlier onset and longer duration of diabetes and probably female sex are considered major contributing factors to disease growth. Future investigations will confirm the usefulness of this diabetic retinopathy animal model.

\footnotetext{
Abbreviations

HFD: High-fat diet; STZ: Streptozotocin; GFAP: Glial-fibrillary-acidic-protein; VEGF: The vascular endothelial growth factor; vWF: Von Willebrand factor; SHBG: Sex hormone binding globulin; ISO: International organization for standardization; H\&E: Haematoxylin and eosin; PAS: Periodic acid-schiff stain SD: Standard deviations; LT-DBT: Long-term diabetic rats; ST-DBT: Short-term diabetic rats; FL: Fibre layer; OPL: Outer plexiform layer.
}

\section{Competing interests}

The authors declare that they have no competing interests.

Authors' contributions

JEM: AB, MT, ES, FG; JOC: MT, FG; JEG: MT, ES, FG. All authors read and approved the final manuscript.

\section{Acknowledgments}

We are grateful to Guillermo Gaston, Soledad Arregui and Norma Montalbetti for their skillful technical assistance. This research and the authors have no commercial interest.

\section{Author details}

Nanomedicine \& Vision Group, Facultad de Ciencias Biomedicas, Department of Ophthalmology, Hospital Universitario Austral, Universidad Austral, Pilar, Buenos Aires, Argentina. ${ }^{2}$ Department of Ophthalmic Pathology,

Fundación Oftalmológica Argentina "Jorge Malbran", Buenos Aires, Argentina.

Received: 23 August 2013 Accepted: 4 November 2013

Published: 13 November 2013

\section{References}

1. Winer N, Sowers JR: Epidemiology of diabetes. J Clin Pharmacol 2004, 44(4):397-405

2. Ginter $\mathrm{E}$, Simko V: Global prevalence and future of diabetes mellitus. Adv Exp Med Biol 2012, 771:35-41.

3. Grossniklaus HE, Kang SJ, Berglin L: Animal models of choroidal and retinal neovascularization. Prog Retin Eye Res 2010, 29(6):500-519.

4. Robinson R, Barathi VA, Chaurasia SS, Wong TY, Kern TS: Update on animal models of diabetic retinopathy: from molecular approaches to mice and higher mammals. Dis Model Mech 2012, 5(4):444-456.

5. Kern TS, Tang J, Berkowitz BA: Validation of structural and functional lesions of diabetic retinopathy in mice. Mol Vis 2010, 16:2121-2131.

6. Ritter MR, Aguilar E, Banin E, Scheppke L, Uusitalo-Jarvinen H, Friedlander M: Three-dimensional in vivo imaging of the mouse intraocular vasculature during development and disease. Invest Ophthalmol Vis Sci 2005, 46(9):3021-3026

7. Friedlander M, Theesfeld CL, Sugita M, Fruttiger M, Thomas MA, Chang S, Cheresh DA: Involvement of integrins alpha $v$ beta 3 and alpha $v$ beta 5 in ocular neovascular diseases. Proc Natl Acad Sci U S A 1996, 93(18):9764-9769.

8. Ding EL, Song Y, Malik VS, Liu S: Sex differences of endogenous sex hormones and risk of type 2 diabetes: a systematic review and metaanalysis. Jama 2006, 295(11):1288-1299.

9. Ferrand N, Astesano A, Phan HH, Lelong C, Rosselin G: Dynamics of pancreatic cell growth and differentiation during diabetes reversion in STZ-treated newborn rats. Am J Physiol 1995, 269(5 Pt 1):C1250-C1264.

10. Villela Dantas A, Zuleica B, de Carvalho Catelli M: Vascular Desease in Diabetic Women: Why Do they Miss the Female Protection? J Diabetes Res 2012, 2012. Article ID 570598, 10 pages.

11. Chen BH, Brennan K, Goto A, Song Y, Aziz N, You NC, Wellons MF, Manson JE, White $D L$, Butch $A W$, et al: Sex hormone-binding globulin and risk of clinical diabetes in American black, Hispanic, and Asian/Pacific Islander postmenopausal women. Clin Chem 2012, 58(10):1457-1466.

12. Ding EL, Song Y, Manson JE, Hunter DJ, Lee CC, Rifai N, Buring JE, Gaziano JM, Liu S: Sex hormone-binding globulin and risk of type 2 diabetes in women and men. N Engl J Med 2009, 361(12):1152-1163.

13. Douglas W, Losordo JM: Inser: estrogen and angiogenesis. Arterioscler Thromb Vasc Biol 2001, 21:6-12

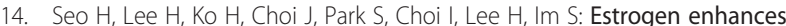
angiogenesis through a pathway involving platelet-activating factormediated nuclear factor-KB activation. Cancer Res 2004, 15(64):6482-6488.

15. Bonner-Weir S, Trent DF, Honey RN, Weir GC: Responses of neonatal rat islets to streptozotocin: limited B-cell regeneration and hyperglycemia. Diabetes 1981, 30(1):64-69.

16. Pascoe WS, Storlien LH: Inducement by fat feeding of basal hyperglycemia in rats with abnormal beta-cell function. Model for study of etiology and pathogenesis of NIDDM. Diabetes 1990, 39(2):226-233.

17. Weir GC, Clore ET, Zmachinski CJ, Bonner-Weir S: Islet secretion in a new experimental model for non-insulin-dependent diabetes. Diabetes 1981, 30(7):590-595.

18. Kuwabara T, Cogan DG: Studies of retinal vascular patterns. I. Normal architecture. Arch Ophthalmol 1960, 64:904-911.

19. Robison WG Jr, McCaleb ML, Feld LG, Michaelis OE, Laver N, Mercandetti M: Degenerated intramural pericytes ('ghost cells') in the retinal capillaries of diabetic rats. Curr Eye Res 1991, 10(4):339-350.

20. Fantus IG, Chayoth R, O'Dea L, Marliss EB, Yale JF, Grose M: Insulin binding and glucose transport in adipocytes in neonatal streptozocin-injected rat model of diabetes mellitus. Diabetes 1987, 36(5):654-660.

21. Srinivasan $\mathrm{K}$, Viswanad B, Asrat $\mathrm{L}$, Kaul CL, Ramarao P: Combination of high-fat diet-fed and low-dose streptozotocin-treated rat: a model for type 2 diabetes and pharmacological screening. Pharmacol Res 2005, 52(4):313-320. 
22. Vital $\mathrm{P}$, Larrieta $\mathrm{E}$, Hiriart $\mathrm{M}$ : Sexual dimorphism in insulin sensitivity and susceptibility to develop diabetes in rats. J Endocrinol 2006, 190(2):425-432.

23. Kobayashi $K$, Kobayashi $H$, Ueda M, Honda Y: Estrogen receptor expression in bovine and rat retinas. Invest Ophthalmol Vis Sci 1998, 39(11):2105-2110.

24. Gupta PD, Johar K Sr, Nagpal K, Vasavada AR: Sex hormone receptors in the human eye. Surv Ophthalmol 2005, 50(3):274-284.

25. De Lorgeril M, Salen P, Martin JM, Monjaud I, Delaye J, Mamelle N: Mediterranean diet, traditional risk factors, and the rate of cardiovascular complications after myocardial infarction: final report of the lion diet heart study. Circulation 1999, 99:779-785.

26. De Lorgeril M, Renaud S, Mamelle N, et al: ,Mediterranean alpha-linoleic acid-rich diet in secondary prevention of coronary heart disease. Lancet 1994, 343:1454-1459.

27. Mancini JE, Ortiz G, Croxatto JO, Gallo JE: Retinal upregulation of inflammatory and proangiogenic markers in a model of neonatal diabetic rats fed on a high-fat-diet. BMC Ophthalmol 2013, 13(1):14.

28. Motiejunaite R, Kazlauskas A: Pericytes and ocular diseases. Exp Eye Res 2008, 86(2):171-177.

29. Tsuji N, Matsuura T, Ozaki K, Sano T, Narama I: Diabetic retinopathy and choroidal angiopathy in diabetic rats (WBN/Kob). Exp Anim 2009, 58(5):481-487.

30. Haurigot V, Villacampa P, Ribera A, Bosch A, Ramos D, Ruberte J, Bosch F: Long-term retinal PEDF overexpression prevents neovascularization in a murine adult model of retinopathy. PLOS ONE 2012, 7(7):e41511.

31. Gariano RF, Iruela-Arispe ML, Sage EH, Hendrickson AE: Immunohistochemical characterization of developing and mature primate retinal blood vessels. Invest Ophthalmol Vis Sci 1996, 37(1):93-103.

32. Vacca A, Ribatti D, Presta M, Minischetti M, lurlaro M, Ria R, Albini A, Bussolino F, Dammacco F: Bone marrow neovascularization, plasma cell angiogenic potential, and matrix metalloproteinase-2 secretion parallel progression of human multiple myeloma. Blood 1999, 93(9):3064-3073.

33. Chen J, Joyal JS, Hatton CJ, Juan AM, Pei DT, Hurst CG, Xu D, Stahl A, Hellstrom A, Smith LE: Propranolol inhibition of beta-adrenergic receptor does not suppress pathologic neovascularization in oxygen-induced retinopathy. Invest Ophthalmol Vis Sci 2012, 53(6):2968-2977.

doi:10.1186/1758-5996-5-68

Cite this article as: Mancini et al:: Proliferative retinopathy and neovascularization of the anterior segment in female type 2 diabetic rats. Diabetology \& Metabolic Syndrome 2013 5:68.

\section{Submit your next manuscript to BioMed Central and take full advantage of:}

- Convenient online submission

- Thorough peer review

- No space constraints or color figure charges

- Immediate publication on acceptance

- Inclusion in PubMed, CAS, Scopus and Google Scholar

- Research which is freely available for redistribution 